\title{
Transmission Electron Microscopy Study of Microstructure and Orientation Relationships in V/Ag Multilayers
}

\author{
Qingmin Wei, Amit Misra
}

Los Alamos National Laboratory, Los Alamos, New Mexico 87545

Due to their unusual properties such as ultra-high strengths, radiation damage tolerance and stabilization of new atomic arrangements at nanometer dimensions, multilayer structures have been the subject of many scientific investigations [1-4]. Microstructures and orientation relationships in sputter-deposited, polycrystalline V/Ag multilayers with different individual thickness ranging from $1 \mathrm{~nm}$ to $50 \mathrm{~nm}$ were investigated. It was found that the wavy morphology of layers resulting from competitive kinetic limitations of deposited atoms gives rise to a variety of orientation relationships: Kurdjumov-Sachs (K-S), Nishiyama-Wasserman (N-W), Bain, Pitsch and many intermediate orientation relationships (OR). In most cases, intermediate orientations with $1^{\circ} \sim 3^{\circ}$ deviations from parallel planes or directions in standard orientations were observed.

Fig. 1 shows the HRTEM analysis of V/Ag multilayers with $10 \mathrm{~nm}$ layer thickness. At the beginning of deposition (Fig. 3a), the V layer grows with its closed packed atomic plane (110) nearly parallel to substrate surface, even though there exists an amorphous layer of native oxide on the Si substrate (Fig. $3 b$ ). At the top or bottom of the curved interfaces, K-S and N-W OR were observed to be dominant (Fig. 3e and f), while at the inclined interfaces, K-S, N-W, Bain, Pitsch and many intermediate OR's were observed. Frequent examples of switching orientations between K-S and N-W at successive interfaces were noted.

Bain orientation was also found in $\mathrm{Ag} / \mathrm{V}$ multilayers (Fig. 2) at an inclined interface where $\{111\} \mathrm{fcc}$ is parallel to $\{110\} \mathrm{bcc}$, and $<110>$ fcc is parallel to $<100>$ bcc. The habit plane is (100) for both layers. In the $\mathrm{V}$ [001] zone axis, two atomic planes (110) and (110) are perpendicular while in the $\mathrm{Ag}$ [011] zone axis, the angle between the two $\{111\}$ planes is $70.5^{\circ}$. Thus for one-to-one correspondence across the interface between the pair of $\{111\} \mathrm{Ag}$ and the pair of $\{110) \mathrm{V}$ planes, the misorientation between a (111) plane with its corresponding (110) plane is $\left(90^{\circ}-70.5^{\circ}\right) / 2=9.75^{\circ}$ (Fig. 2b), in good agreement with experimental observations (Fig. 2a). The edge components of the misfit dislocations at the interface are also noted in the HRTEM images (Fig. 2c). These dislocations can be divided into two sets along two perpendicular directions. In contrast to normal tilt boundaries in which the sign of dislocation in each set is the same, $\mathrm{V} / \mathrm{Ag}$ interface shows that adjacent dislocations in a given set have opposite signs. The average dislocation spacing within each set is about $5 \mathrm{~nm}$. Fig. 3 shows the bubble distribution in V/Ag multilayers after $33 \mathrm{keV}$ He implantation up to dose of $1 \times 10^{17} \mathrm{~cm}^{-2}$ at room temperature. It can be seen that detectable bubbles are only in Ag layers.

\section{References}

[1] Misra A, Hirth JP, Hoagland RG, Acta Mater 53 (2005) 4817.

[2] Grimes RW, Konings RJM, Edwards L, Nature Mater 7 (2008) 683.

[3] Demkowicz MJ, Hoagland RG, Hirth JP, Phys Rev Lett 100 (2008) 136102.

[4] Li QZ, Anderson, PM, 53 (2005) 1121. 

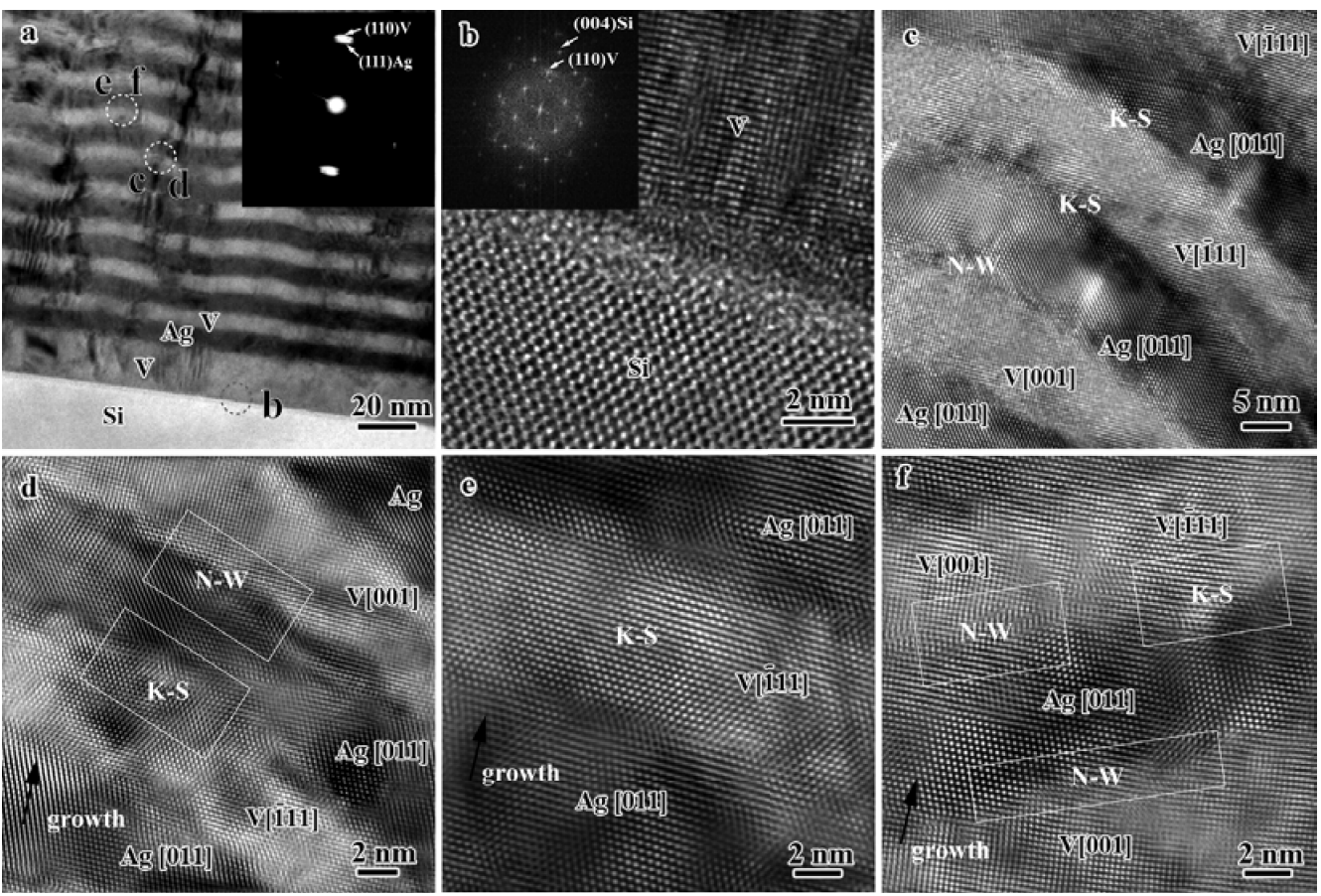

FIG. 1: Cross-sectional TEM images. (a) Morphology of multilayers. Inset shows SAED pattern. Dashed circles indicate the location where images (b), (c), (d), (e) and (f) are taken. (b) Interface structure between $\mathrm{V}$ and substrate Si. Insets show FFT spectrum. (c) And (d) HRTEM image showing K-S and N-W orientation relationships. (e) And (f) K-S and N-W orientation relationships.
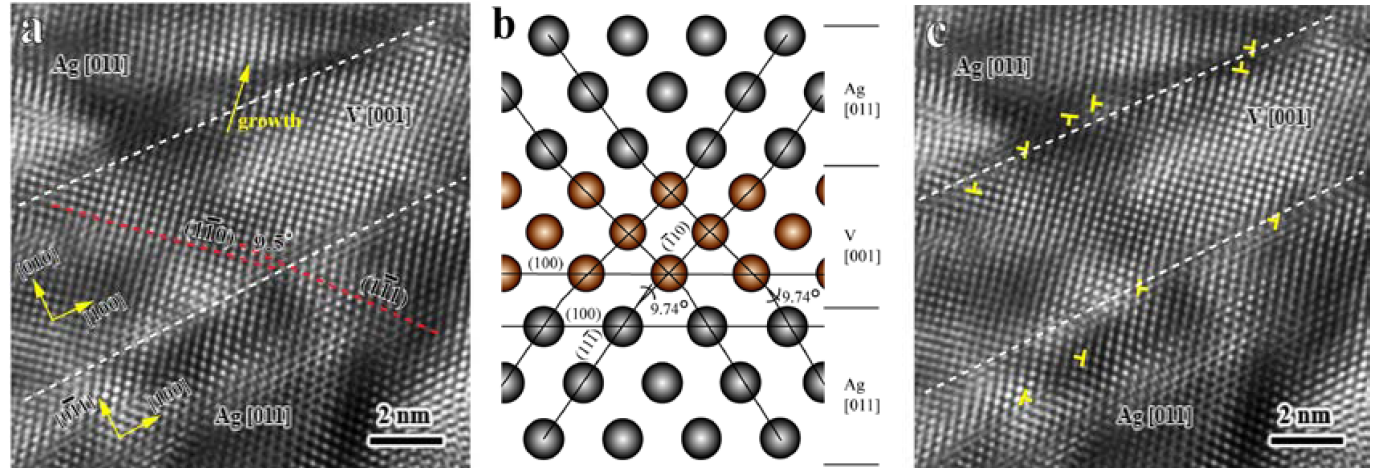

FIG. 2: Bain orientation observed in V-Ag multilayers: (a) experimental image and (b) schematic illustration. (c) Two sets of dislocations.
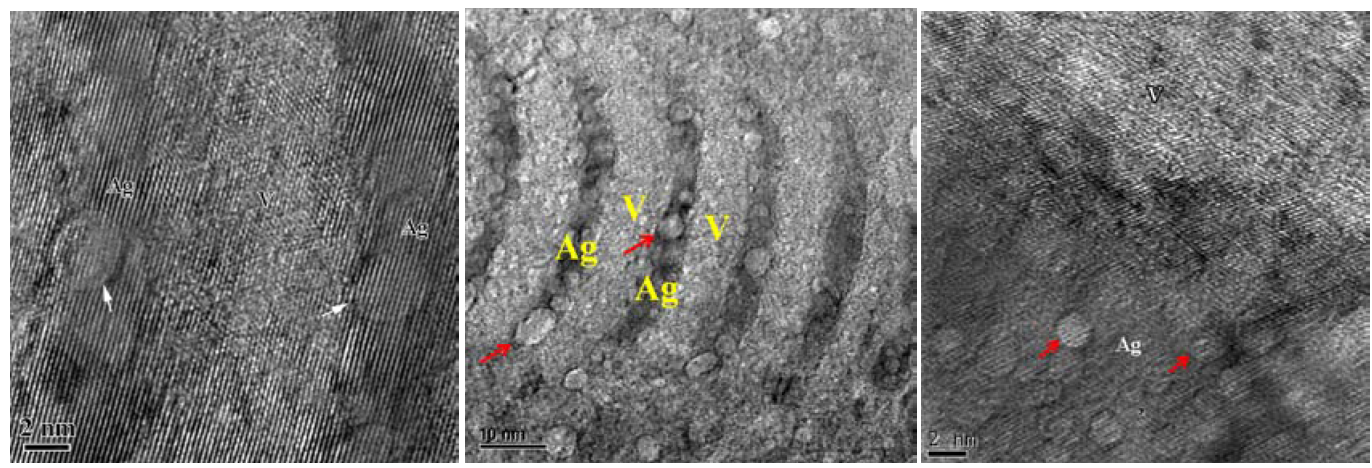

FIG. 3: Bubble formation at (a) $5 \mathrm{~nm}$ V/Ag layer, (b) $10 \mathrm{~nm} \mathrm{V/Ag} \mathrm{layer} \mathrm{,} \mathrm{and} \mathrm{(c)} 50 \mathrm{~nm}$ $\mathrm{V} / \mathrm{Ag}$ layer. Arrows indicate bubbles. 